\title{
Burden and risk factors for Pseudomonas aeruginosa community-acquired pneumonia: a multinational point prevalence study of hospitalised patients
}

\author{
Marcos I. Restrepo ${ }^{1,2}$, Bettina L. Babu ${ }^{1,2}$, Luis F. Reyes ${ }^{3,4}$, James D. Chalmers ${ }^{5}$, \\ Nilam J. Soni ${ }^{1,2}$, Oriol Sibila $\mathbb{1}^{6}$, Paola Faverio ${ }^{7}$, Catia Cilloniz $\mathbb{1}^{8}$, \\ William Rodriguez-Cintron ${ }^{9}$ and Stefano Aliberti (i) $^{10}$
}

\begin{abstract}
Affiliations: ${ }^{1}$ Division of Pulmonary Diseases and Critical Care Medicine, University of Texas Health - San Antonio, San Antonio, TX, USA. ${ }^{2}$ Division of Pulmonary Diseases and Critical Care Medicine, South Texas Veterans Health Care System, San Antonio, TX, USA. ${ }^{3}$ Dept of Microbiology, Universidad de la Sabana, Chía, Colombia. ${ }^{4}$ Dept of Critical Care Medicine, Clinica Universidad de La Sabana, Chía, Colombia. ${ }^{5}$ School of Medicine, University of Dundee, Dundee, UK. ${ }^{6}$ Servei de Pneumologia, Departamento de Medicina, Hospital Santa Creu i Sant Pau, Universitat Autònoma de Barcelona, Barcelona, Spain. ${ }^{7}$ Cardio-Thoracic-Vascular Dept, University of Milan Bicocca, Respiratory Unit, San Gerardo Hospital, ASST di Monza, Monza, Italy. ${ }^{8}$ Dept of Pneumology, Institut Clinic del Tórax, Hospital Clinic of Barcelona - Institut d'Investigacions Biomèdiques August Pi i Sunyer (IDIBAPS), University of Barcelona (UB), CIBER, Barcelona, Spain. ${ }^{9}$ Pulmonary/Critical Care Medicine VA Caribbean Healthcare System, San Juan, Puerto Rico. ${ }^{10}$ Dept of Pathophysiology and Transplantation, University of Milan, Cardio-thoracic unit and Adult Cystic Fibrosis Centre, Fondazione IRCCS Ca' Granda Ospedale Maggiore Policlinico, Milan, Italy.
\end{abstract}

Correspondence: Marcos I. Restrepo, South Texas Veterans Health Care System ALMD, 7400 Merton Minter Boulevard, San Antonio, TX, 78229, USA. E-mail: restrepomauthscsa.edu

@ERSpublications

$P$. aeruginosa is infrequent in CAP patients. Specific risk factors should be assessed when choosing antibiotics for CAP. http://ow.ly/jpP130koSMX

Cite this article as: Restrepo MI, Babu BL, Reyes LF, et al. Burden and risk factors for Pseudomonas aeruginosa community-acquired pneumonia: a multinational point prevalence study of hospitalised patients. Eur Respir J 2018; 52: 1701190 [https://doi.org/10.1183/13993003.01190-2017].

ABSTRACT Pseudomonas aeruginosa is a challenging bacterium to treat due to its intrinsic resistance to the antibiotics used most frequently in patients with community-acquired pneumonia (CAP). Data about the global burden and risk factors associated with $P$. aeruginosa-CAP are limited. We assessed the multinational burden and specific risk factors associated with $P$. aeruginosa-CAP.

We enrolled 3193 patients in 54 countries with confirmed diagnosis of CAP who underwent microbiological testing at admission. Prevalence was calculated according to the identification of $P$. aeruginosa. Logistic regression analysis was used to identify risk factors for antibiotic-susceptible and antibiotic-resistant $P$. aeruginosa-CAP.

The prevalence of $P$. aeruginosa and antibiotic-resistant $P$. aeruginosa-CAP was $4.2 \%$ and $2.0 \%$, respectively. The rate of $P$. aeruginosa CAP in patients with prior infection/colonisation due to $P$. aeruginosa and at least one of the three independently associated chronic lung diseases (i.e. tracheostomy, bronchiectasis and/or very severe chronic obstructive pulmonary disease) was $67 \%$. In contrast, the rate of $P$. aeruginosa-CAP was $2 \%$ in patients without prior $P$. aeruginosa infection/colonisation and none of the selected chronic lung diseases.

The multinational prevalence of $P$. aeruginosa-CAP is low. The risk factors identified in this study may guide healthcare professionals in deciding empirical antibiotic coverage for CAP patients.

This article has supplementary material available from erj.ersjournals.com

Received: June 152017 | Accepted after revision: June 062018

The content of this work is not subject to copyright. Design and branding are copyright @ERS 2018 


\section{Introduction}

Community acquired pneumonia (CAP) is a leading infectious cause of morbidity and mortality worldwide [1, 2]. 5-6 billion people are diagnosed with CAP and >3.5 million people die annually secondary to CAP [1,3]. Several viruses and bacteria cause CAP [4], but Streptococcus pneumoniae remains the most frequently identified bacterial pathogen in adults [5]. During the past few decades, the aetiology of CAP has been changing: antibiotic-resistant bacteria that were thought to be important only in hospital settings are now becoming more prevalent in community settings [6-8]. The evolution of this new pathogen ecology is threatening our capacity to treat patients with CAP [9-11].

Pseudomonas aeruginosa, a Gram-negative bacterium, intrinsically resistant to several groups of antibiotics, such as $\beta$-lactams [12], has been reported frequently in CAP patients with specific healthcare associated risk factors, such as residence in a nursing home and hospitalisation during the past 90 days (i.e. healthcare-associated pneumonia (HCAP)) [13-15]. Moreover, severe illnesses and poor clinical outcomes have been linked to P. aeruginosa infection in patients with CAP [15-17]. During recent years, circulating strains of $P$. aeruginosa have tended to have higher resistance patterns to antipseudomonal antibiotics, leading to infections that are challenging to treat [6].

The prevalence of CAP due to $P$. aeruginosa varies significantly between different patient groups and specific risk factors for $P$. aeruginosa-CAP are controversial $[18,19]$. Currently available data about the prevalence of $P$. aeruginosa-CAP and its resistance patterns are limited to single-centre studies $[15,20]$ and studies with several methodological limitations [15, 21-23]. In a meta-analysis, Chalmers et al. [24] reported data from 22 studies a prevalence ranging from $0 \%$ to $23 \%$ in different CAP populations and a pool prevalence of $8.6 \%$ for $P$. aeruginosa in patients with multidrug-resistant (MDR) risk factors and $4 \%$ in patients without risk factors. All were single-centre/-region and the majority were rated as having poor methodological status [24]. As a result, the true prevalence of $P$. aeruginosa-CAP globally is unknown. Currently, the risk factors associated with $P$. aeruginosa infection recognised by Infectious Diseases Society of America (IDSA)/American Thoracic Society (ATS) guidelines for HCAP differ from those published in the CAP guidelines $[13,25]$. Furthermore, the global prevalence of MDR $P$. aeruginosa in CAP patients is unknown. Therefore, to close the gap regarding the global prevalence and the risk factors associated with $P$. aeruginosa, we designed a multinational, multicentre, worldwide study to determine the point prevalence of $P$. aeruginosa, its antibiotic resistance patterns and the associated risk factors in hospitalised patients with CAP.

\section{Materials and methods}

\section{Study design}

This is a multicentre, point-prevalence study of hospitalised patients with CAP in 222 hospitals in 54 countries. The coordinating institution for this project was the University of Texas Health San Antonio (UT Health) (San Antonio, TX, USA). The UT Health institutional review board approved the project to collect data and serve as a coordinating institution for the study (\#HSC20150184E). Study sites had to comply with local and national research regulations to participate in the study. Electronic invitations to participate were sent to members of various medical professional societies representing physicians specialising in infectious diseases, pulmonary, critical care and internal medicine. Additionally, first authors of previously published studies of MDR pathogens in CAP were sent individual invitations. This project received no funding. Subjects were enrolled on four randomly selected days in March, April, May and June 2015. Each site investigator selected the study days to participate in compliance with the IRB stipulations, and avoid potential patient de-identification [26]. Due to the observational nature of the study, patient consent was not required.

\section{Study subjects}

Inclusion criteria

Patients aged $\geqslant 18$ years hospitalised with CAP were eligible for the study. CAP had to be diagnosed per the current IDSA/ATS guidelines [13]: presence of new pulmonary infiltrates on thoracic imaging (chest radiograph, computed chest tomography or lung ultrasound) during the initial $48 \mathrm{~h}$ of hospitalisation and one or more of the following conditions: 1) novel or increased cough with or without sputum production and/or purulent respiratory secretions; 2 ) fever (oral or rectal temperature $\geqslant 37.8^{\circ} \mathrm{C}$ ) or hypothermia (oral or rectal temperature $<36^{\circ} \mathrm{C}$ ); 3) signs of systemic inflammation (abnormal white blood cell count (leukocytosis $>10000$ cells $\cdot \mathrm{cm}^{-3}$, bandemia $>10 \%$, leukopaenia $<4000 \mathrm{cells} \cdot \mathrm{cm}^{-3}$ ), procalcitonin levels above the local upper limit of normal or increased C-reactive protein) [26].

\section{Exclusion criteria}

Patients were excluded from participating in the study if they had hospital-acquired pneumonia (HAP) or ventilator associated pneumonia (VAP) [25]. In addition, patients without microbiological testing from 
either blood, lower respiratory tract cultures or sputum collected within $24 \mathrm{~h}$ of hospital admission were excluded.

\section{Data collection}

Data was collected and managed using REDCap ${ }^{\mathrm{TM}}$ (Research Electronic Data Capture), an electronic data capture tool hosted on the UT Health San Antonio server. REDCap ${ }^{\mathrm{TM}}$ is a protected web-based application designed to collect research data [27]. Confirmation of microbiological results and all electronic data entry had to be completed within 7 days of study enrolment.

\section{Microbiological analysis}

Diagnostic testing, such as blood cultures and respiratory collection, and clinical care decisions were decided by attending physicians, not per study protocol [26]. Local microbiological testing protocols were used to process blood and sputum samples collected within the first $24 \mathrm{~h}$ of hospitalisation. If available, data on pleural fluid, tracheobronchial aspirate and bronchoalveolar lavage fluid were collected. Local testing executed by each hospital included respiratory and blood cultures, urinary antigen and drug susceptibility testing. Local quality control protocols for minimum inhibitory concentration breakpoints and Clinical and Laboratory Standards Institute (CLSI) or the European Committee on Antimicrobial Susceptibility Testing (EUCAST) standards were followed according to local regulations and protocols $[28,29]$.

\section{Study definitions}

Patients that required invasive respiratory and/or vasopressor support (IRVS) during the initial $24 \mathrm{~h}$ of hospitalisation were categorised as IRVS $^{+}$[30]. P. aeruginosa-CAP was defined as any patient with a confirmed diagnosis of CAP in whom $P$. aeruginosa was isolated in any respiratory fluid, including pleural effusion, sputum and/or bronchoalveolar lavage and/or in the blood. Drug-resistant $P$. aeruginosa was defined when the isolated pathogen was resistant to at least one antibiotic and MDR P. aeruginosa was defined by resistance to at least three of the evaluated antibiotics. Antibiotic resistance to the following antipseudomonal antibiotics were evaluated: piperacillin/tazobactam, cefepime, ceftazidime, amikacin, gentamicin, tobramycin, levofloxacin, ciprofloxacin, imipenem, meropenem, doripenem, colistin and polymyxin B.

Prior $P$. aeruginosa infection/colonisation was defined as confirmed infection/colonisation within the past year before the hospitalisation, documented by the patient or available patient records. Chronic obstructive pulmonary disease (COPD) was defined according to forced expiratory volume in $1 \mathrm{~s}$ (FEV1)/forced vital capacity ratio $<0.7$ and a compatible clinical history (including smoking history if relevant). Very severe COPD was defined as patients with COPD with evidence of very severe obstruction, determined by FEV 1 $<30 \%$ prior to hospital admission (a complete study dictionary can be found in the online supplementary material). Site investigators were given these definitions in the study protocol manual and study dictionary prior to starting data collection [26].

\section{Statistical analysis}

The prevalence of $P$. aeruginosa as microbiological aetiology of CAP (i.e. P. aeruginosa-CAP) was calculated based on the counts of $P$. aeruginosa isolates divided by the study cohort (i.e. CAP patients with completed microbiological test) and is expressed as a percentage. Chi-squared tests were used to compare categorical variables, which were expressed as $\mathrm{n}(\%)$. Continuous variables are presented as median (interquartile range (IQR)) and the nonparametric Mann-Whitney U-test was used to compare them. To assess the relationship between CAP due to P. aeruginosa and 67 demographic, clinical, epidemiological and treatment variables, a stepwise logistic regression model was executed. A p-value $<0.05$ was defined as statistically significant. Tableau Desktop, Professional for Mac (Seattle, WA, USA), was used to generate prevalence maps. All statistical analyses were performed with IBM SPSS, Statistics for Mac (version 22.0; IBM, Armonk, NY, USA).

\section{Results}

A total of 3193 patients were recruited during four study days from 222 hospitals in 54 countries ( $\mathrm{n}=1877$ (58.8\%) male, median (IQR) age 68 (54-80) years). Demographics, risk factors, comorbidities, severity of illness and chronic treatments are reported in table 1. Among the six continents, most patients were recruited from Europe $(n=1941,60.8 \%)$, followed by North America $(n=484,15.2 \%)$, Asia $(n=405,12.7 \%)$, South America $(n=203,6.4 \%)$, Africa $(n=128,4.0 \%)$ and Oceania $(n=32,1.0 \%)$. Microbiological cultures were obtained from blood $(n=2211,69.2 \%)$, sputum $(n=1630,51.0 \%)$ and bronchoalveolar lavage $(n=311,9.7 \%)$. At least one pathogen was identified in 1173 patients (36.7\%) (online supplementary figure E1). Table 2 and online supplementary table E1 display the prevalence of $P$. aeruginosa-CAP and antibiotic-resistant $P$. aeruginosa-CAP per continent and country. 
TABLE 1 Characteristics of patients with Pseudomonas aeruginosa community-acquired pneumonia (CAP) versus non- $P$. aeruginosa-CAP.

\section{CAP}

3193

\section{Subjects}

Demographic characteristics

Age years

Male

Underweight $\mathrm{n} / \mathrm{N}(\%)$

Obese

Respiratory past medical history

Active lung cancer

Asthma

Bronchiectasis

Chronic aspiration

COPD

Very severe COPD (FEV $1 \leqslant 30 \%$ )

Current/former smoker

Interstitial lung disease

Obstructive sleep apnoea

Oxygen therapy at home

Lung transplantation

Tracheostomy

Cardiovascular past medical history

Arrhythmia

Coronary artery disease

Heart failure

Hypertension

Stroke

Chronic medication

Inhaled corticosteroids

Proton pump inhibitor

Statins

Steroids

Chronic interventions

Enteric tube feeding

Haemodialysis

Indwelling catheter

\section{Immunosuppressive conditions}

Active solid tumour

AIDS

Aplastic anaemia

Asplenia

Biological drug use

Chemotherapy in the past 3 months

Haematological malignancy

HIV infection

Immunocompromised patients

Neutropenia

Other immunosuppressive condition

Other chronic medical conditions

Chronic renal failure

Dementia

Diabetes mellitus

Liver disease

Malnutrition

Mental illness

Prosthetic material

Recurrent skin infections

Other, nonmedical conditions

Bedridden

Contact sport

Healthcare worker

Homeless

P. aeruginosa-CAP

133

Non- $P$. aeruginosa-CAP

p-value

\section{$68(54-80)$ \\ 1877 (58.8) \\ $150 / 2055$ (7.3) \\ $510(16)$}

92 (2.9)

234 (7.3)

168 (5.3)

218 (6.8)

834 (26.1)

$90(2.8)$

1114 (34.9)

91 (2.8)

123 (3.9)

208 (6.5)

$7(0.2)$

$50(1.6)$

455 (14.2)

526 (16.5)

418 (13.1)

1444 (45.2)

250 (7.8)

544 (17)

907 (28.4)

$670(21)$

268 (8.4)

48 (1.5)

$51(1.6)$

67 (2.1)

245 (7.7)

57 (1.8)

$13(0.4)$

$12(0.4)$

35 (1.1)

$134(4.2)$

150 (4.70)

107 (3.4)

623 (19.5)

44 (1.4)

125 (3.9)

349 (10.9)

333 (10.4)

681 (21.3)

129 (4)

289 (9.1)

$220(0.726)$

$100(3.1)$

55 (1.7)

353 (11.1)

5 (0.2)

44 (1.4)

31 (1)

$64.36(52.5-78.5)$
$79(59.4)$
$9 / 89(10.1)$
$17(12.8)$

5 (3.8)

$8(6)$

31 (23.3)

15 (11.3)

57 (42.9)

16 (12.0)

46 (34.6)

6 (4.5)

6 (4.5)

26 (19.5)

$1(0.8)$

15 (11.3)

20 (15)

33 (24.8)

25 (18.8)

62 (46.6)

$13(5.2)$

$48(36.1)$

$58(6.4)$

37 (27.8)

21 (15.8)

\section{8 (6)}

4 (3)

11 (8.3)

11 (4.5)

1 (0.8)

0 (0)

0 (0)

3 (2.3)

4 (3)

3 (2.3)

3 (2.3)

28 (21.1)

1 (0.8)

11 (8.3)

15 (11.3)

14 (10.5)

28 (4.1)

4 (3)

20 (15)

10 (7.5)

2 (1.5)

4 (3.0)

28 (21.1)

0 (0)

4 (3)

0 (0)
3060

$\begin{array}{cc}65.01(54-80) & 0.748 \\ 1798(58.8) & 0.883 \\ 141 / 1966(7.2) & 0.297 \\ 493(16.1) & 0.305\end{array}$

87 (2.8)

0.536

226 (7.4)

137 (4.5)

$203(6.6)$

777 (25.4)

74 (2.4)

1068 (34.9)

85 (2.8)

117 (3.8)

182 (5.9)

6 (0.2)

35 (1.1)

0.553

$<0.001$

0.038

$<0.001$

$<0.001$

0.940

0.240

0.687

$<0.001$

0.180

$<0.001$

435 (14.2)

0.791

493 (16.1)

0.008

0.046

0.742

0.394

237 (7.7)

$<0.001$

496 (16.2)

849 (27.7)

633 (20.7)

268 (8.4)

$<0.001$

0.048

0.002

40 (1.3)

$<0.001$

$47(1.5)$

$56(1.8)$

0.185

$<0.001$

$234(7.6)$

0.791

56 (1.8)

0.358

0.451

$12(0.4)$

0.469

32 (1)

0.190

130 (4.2)

0.485

147 (4.8)

0.174

104 (3.4)

0.473

595 (19.4)

0.647

43 (1.4)

0.527

114 (3.7)

0.008

334 (10.9)

0.895

319 (10.4)

0.970

653 (21.3)

0.937

125 (4.1)

0.537

269 (8.8)

0.014

$210(6.9)$

0.770

98 (3.2)

0.271

51 (1.7)

0.245

325 (92.1)

$<0.001$

5 (0.2)

0.641

40 (1.3)

0.100

0.243 
TABLE 1 Continued

\begin{tabular}{|c|c|c|c|c|}
\hline & CAP & P. aeruginosa-CAP & Non-P. aeruginosa-CAP & p-value \\
\hline Injection of illicit drugs & $37(1.2)$ & $1(0.8)$ & $36(1.2)$ & 0.654 \\
\hline Living in crowded conditions & $671(21)$ & $24(18)$ & $647(21.1)$ & 0.391 \\
\hline Worker in livestock meat industry & $29(0.9)$ & $0(0)$ & $29(0.9)$ & 0.259 \\
\hline \multicolumn{5}{|l|}{ Previous infection/colonisation } \\
\hline Prior MRSA infection/colonisation & $81(2.5)$ & $10(7.5)$ & $71(2.3)$ & $<0.001$ \\
\hline \multicolumn{5}{|l|}{ Prior healthcare exposure (during the past 12 months) } \\
\hline Antibiotic infusion at home & $140(4.4)$ & 12 (9) & $128(4.2)$ & 0.008 \\
\hline Emergency room admission & $972(30.4)$ & $63(47.4)$ & $909(29.7)$ & $<0.001$ \\
\hline Hospitalisations & $1026(32.1)$ & $70(52.6)$ & $956(31.2)$ & $<0.001$ \\
\hline Intravenous antibiotics & $812(25.4)$ & $64(48.1)$ & $748(24.4)$ & $<0.001$ \\
\hline LRTI & $928(29.1)$ & $66(49.6)$ & $862(28.5)$ & $<0.001$ \\
\hline Noninvasive mechanical ventilation & $334(10.5)$ & 15 (11.3) & $319(10.4)$ & 0.753 \\
\hline
\end{tabular}

Data are presented as $\mathrm{n}$, median (interquartile range) or $\mathrm{n}(\%)$, unless otherwise stated. COPD: chronic obstructive pulmonary disease; FEV1: forced expiratory volume in $1 \mathrm{~s}$; MRSA: methicillin-resistant Staphylococcus aureus; ESBL: extended-spectrum $\beta$-lactamases; LRTI: lower respiratory tract infection; ICU: intensive care unit.

Prevalence of $\mathrm{P}$. aeruginosa-CAP

P. aeruginosa-CAP was identified in 133 (4.2\%) patients, representing 11.3\% (133 out of 1173) of all patients who had a positive culture for bacterial pathogens. The continental prevalence of $P$. aeruginosa-CAP was 3.8\% in Europe, $4.3 \%$ in North America, 5.2\% in Asia, 4.9\% in South America, 5.5\% in Africa and 3.1\% in Oceania (figure 1). The prevalence of $P$. aeruginosa-CAP in each country, and a comparison between continents and countries is shown in table 2 and online supplementary table E1. No statistically significant difference was found in the prevalence of $P$. aeruginosa-CAP among different continents. Croatia was the only country with statistically significant higher prevalence of $P$. aeruginosa-CAP (online supplementary table E1).

Prevalence of antibiotic-resistant $\mathrm{P}$. aeruginosa-CAP

Antibiotic-resistant $P$. aeruginosa-CAP was identified in $64(2.0 \%)$ patients, representing 5.4\% (64 out of 1173 ) of all patients who had a positive culture. The continental prevalence of antibiotic-resistant P. aeruginosa-CAP was $1.6 \%$ in Europe, $2.5 \%$ in North America, 2.2\% in Asia, $3.0 \%$ in South America, and $3.9 \%$ in Africa (figure 1). There were no patients with antibiotic-resistant $P$. aeruginosa-CAP identified in Oceania. No statistically significant difference was found in the prevalence of antibiotic-resistant P. aeruginosa-CAP among different continents (table 2).

\section{Prevalence of MDR P. aeruginosa-CAP}

MDR P. aeruginosa was identified in 33 (1.0\%) patients, representing 2.8\% (33 out of 1173) of all patients who had a positive culture. The continental prevalence of MDR P. aeruginosa-CAP was $0.9 \%$ in Europe, $1.2 \%$ in North America, $0.5 \%$ in Asia, $2 \%$ in South America and 2.3\% in Africa. There were no patients with MDR $P$. aeruginosa identified in Oceania. No statistically significant difference was found in the prevalence of MDR $P$. aeruginosa-CAP among different continents. No patients were infected with pan-drug resistant $P$. aeruginosa (i.e. resistant to three or more groups of antibiotics and colistin) [31].

\section{Risk factors}

Patient demographics and risk factors were compared among $P$. aeruginosa-CAP and non- $P$. aeruginosa CAP (table 1). The risk factors independently associated with $P$. aeruginosa-CAP in the multivariate analysis were prior Pseudomonas infection/colonisation (OR 16.10, 95\% CI 9.48-27.35), prior tracheostomy (OR 6.50, 95\% CI 2.61-16.19), bronchiectasis (OR 2.88, 95\% CI 1.65-5.05), IRVS (OR 2.33, 95\% CI 1.44-3.78) and very severe COPD (OR 2.76, 95\% CI 1.25-6.06) (table 3, online supplementary figure E2). Moreover, the risk factors associated with antibiotic-resistant $P$. aeruginosa-CAP were prior Pseudomonas infection/colonisation (OR 17.29, 95\% CI 9.95-33.42), tracheostomy (OR 5.55, 95\% CI 1.73- 
TABLE 2 Prevalence of antibiotic-resistant Pseudomonas aeruginosa-community-acquired pneumonia (CAP) and antibiotic-resistant $P$. aeruginosa-CAP in participating centres representing different continents

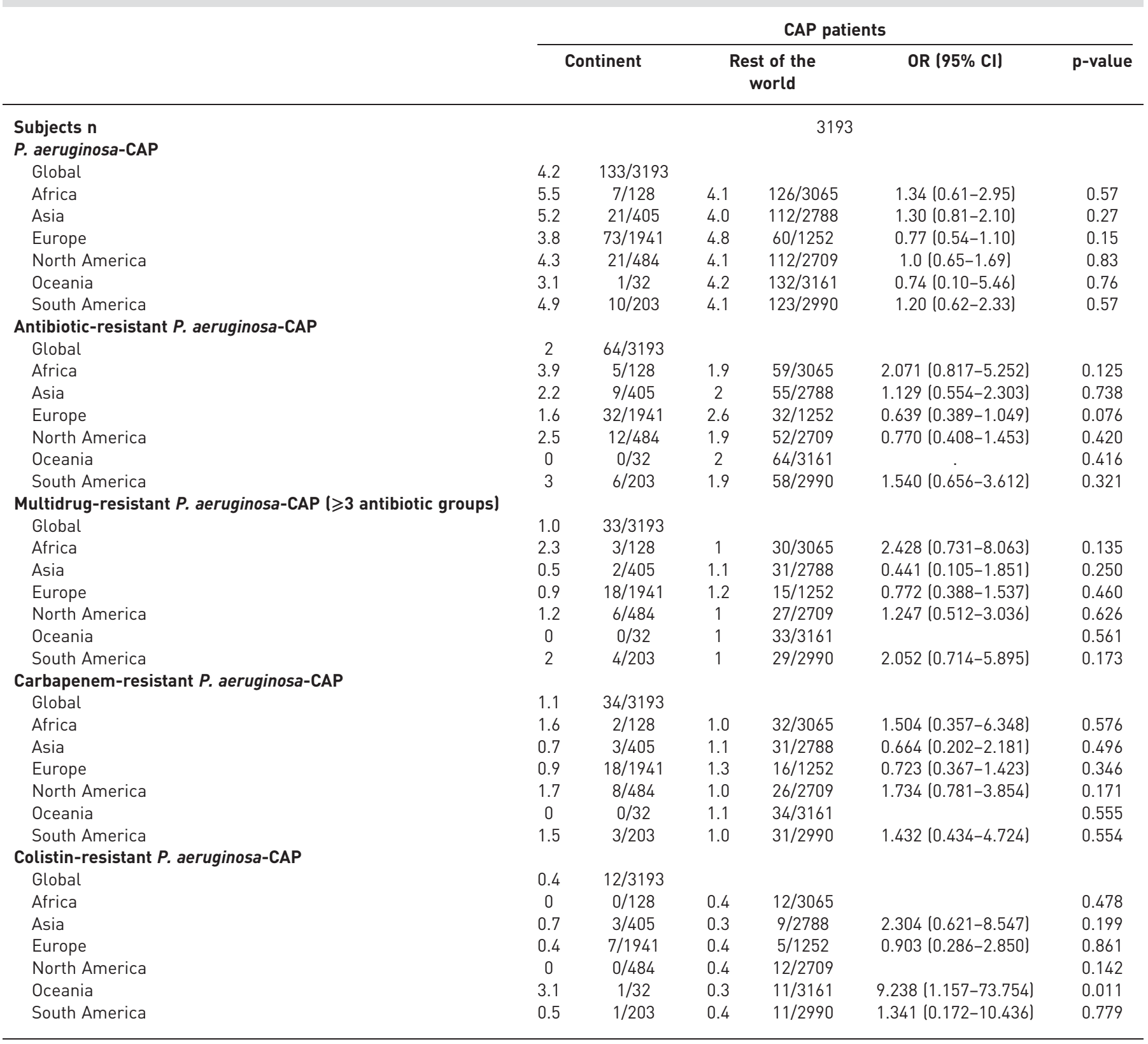

Data are presented as $\%$ or $\mathrm{n} / \mathrm{N}$, unless otherwise stated.

17.80) and IRVS (OR 3.12, 95\% CI 1.63-5.97). The risk factors found to be only statistically significant in the bivariate analysis were COPD, coronary artery disease, inhaled corticosteroid use, indwelling catheter and lower respiratory tract infection/emergency room visits/hospitalisations/antibiotic treatments during the past 12 months (online supplementary table E3). Table 3 shows all the risk factors that had a statistically significant association with the different antibiotic resistance patterns (multivariate analyses are presented in online supplementary table E2).

Figure 2 emphasises the importance of the prevalence of $P$. aeruginosa-CAP, either one of the two main clusters of risk factors were tested among all the patients hospitalised with CAP or after stratifications based on the requirement of IRVS. Among all the patients with CAP, only $11 \%$ had risk factors (previous infection/colonisation by Pseudomonas and had chronic lung diseases (i.e. tracheostomy, bronchiectasis and/or very severe COPD). The lack of these two sets of risk factors (i.e. previous infection/colonisation by Pseudomonas and tracheostomy, bronchiectasis and/or very severe COPD based on FEV1) confirms that 
a)

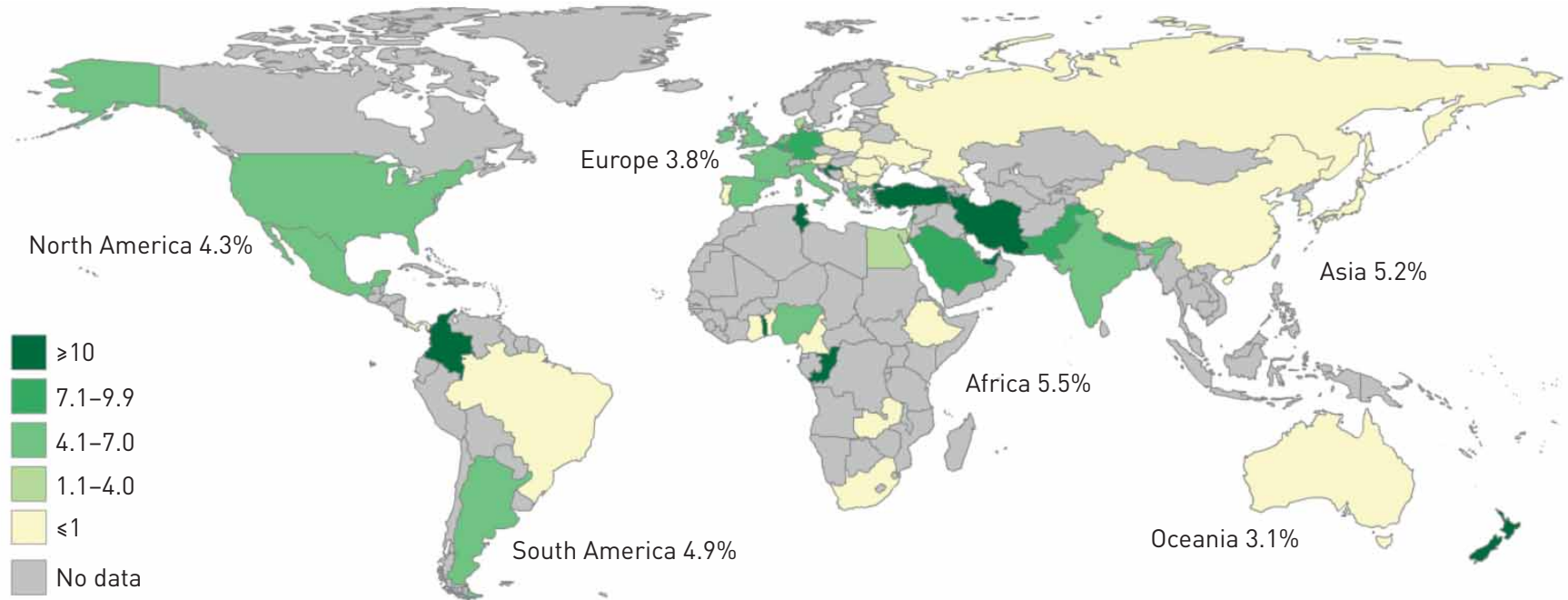

b)

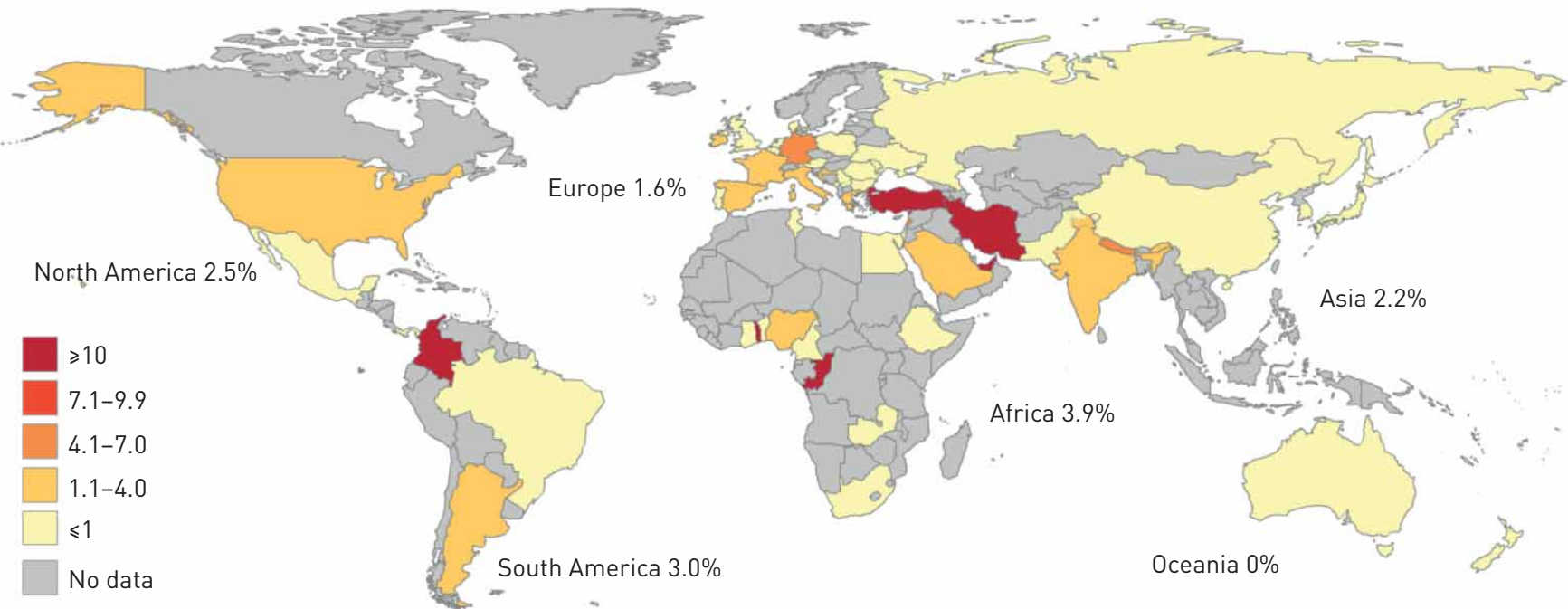

FIGURE 1 Prevalence of a) Pseudomonas aeruginosa-community-acquired pneumonia (CAP) and b) antibiotic-resistant $P$. aeruginosa-CAP by continent.

only $2-6 \%$ of patients might still be affected, independent of the need for IRVS. The sensitivity, specificity, positive/negative predictive values and positive/negative likelihood ratios of these risk factors are presented in online supplementary table E3.

\section{Discussion}

This multinational point-prevalence study found a low prevalence of $P$. aeruginosa among hospitalised patients with CAP. Only $4.2 \%, 2.0 \%$ and $1.0 \%$ of hospitalised patients had CAP due to $P$. aeruginosa, antibiotic-resistant $P$. aeruginosa or multidrug-resistant (MDR) $P$. aeruginosa, respectively. No patients were identified with pan-drug resistant $P$. aeruginosa. There was no statistically significant difference in prevalence rates between different continents. Croatia was the only country with statistically significant higher P. aeruginosa-CAP prevalence. Prior Pseudomonas infection/colonisation, tracheostomy, bronchiectasis, IRVS and very severe COPD were independently associated with $P$. aeruginosa-CAP. Moreover, patients admitted with CAP with a past medical history of Pseudomonas infection/colonisation or chronic lung diseases (i.e. tracheostomy, bronchiectasis and/or very severe COPD) had a higher risk of being infected with antibiotic-resistant $P$. aeruginosa.

Deciding empirical treatment for CAP patients has become challenging due to the emerging prevalence of drug-resistant bacteria, such as $P$. aeruginosa [6]. In a large retrospective study of culture-positive patients 
TABLE 3 Multivariate analysis of risk factors for Pseudomonas aeruginosa-community-acquired pneumonia (CAP), antibiotic-resistant $P$. aeruginosa-CAP, multidrug-resistant (MDR) $P$. aeruginosa-CAP and specific antibiotic resistance patterns

\begin{tabular}{lcccccc} 
& Subjects & Prior $P$. aeruginosa & IRVS & Tracheostomy & Bronchiectasis & COPD \\
\hline P. aeruginosa-CAP & 133 & $16.10(9.48-27.35)$ & $2.33(1.44-3.78)$ & $6.50(2.61-16.19)$ & $2.88(1.65-5.05)$ & \\
Antibiotic-resistant $P$. aeruginosa-CAP & 64 & $17.29(9.95-33.42)$ & $3.12(1.63-5.97)$ & $5.55(1.73-17.80)$ & & $2.76(1.25-6.06)$ \\
Anti-pseudomonal cephalosporins & 38 & $17.79(7.32-43.22)$ & & & \\
Piperacillin/tazobactam & 30 & $9.72(3.88-24.36)$ & $4.14(1.75-9.81)$ & & $3.33(1.21-9.19)$ & \\
Carbapenems & 34 & $10.62(1.26-26.45)$ & $2.70(1.14-6.34)$ & $10.77(3.09-37.52)$ & & \\
Aminoglycosides & 31 & $17.32(7.21-41.61)$ & $3.02(1.24-7.31)$ & & \\
Quinolones & 50 & $17.35(8.28-36.38)$ & $2.84(1.39-5.78)$ & $4.35(1.21-15.60)$ & & \\
MDR P. aeruginosa-CAP & 33 & $12.34(5.05-30.14)$ & $3.42(1.47-7.97)$ & & $2.69(1.10-6.55)$ & \\
\hline
\end{tabular}

Data are presented as $\mathrm{n}$ or OR $(95 \% \mathrm{CI})$, unless otherwise stated. IRVS: invasive respiratory or vasopressor support; COPD: chronic obstructive pulmonary disease.

in the United States, Kollef et al. [15] reported a 19\% prevalence of P. aeruginosa in hospitalised patients with CAP. In a prospective cohort study in Europe, CHALMERs et al. [32] reported a $0.7 \%$ prevalence of $P$. aeruginosa among all patients enrolled with CAP and $2.2 \%$ among those with positive cultures. JAIN et al. [5] found $P$. aeruginosa in $0.4 \%$ of patients with CAP in a prospective cohort study in the United States and $1 \%$ among the patients with culture-positive pneumonia. We found the multinational point prevalence of $P$. aeruginosa as the causative pathogen of CAP was $4.2 \%$, which corresponds to $11.3 \%$ of patients with positive-culture pneumonia. Variations in the prevalence rates reported by different studies may be explained by differences in study design, especially the denominator used to calculate the prevalence rates [26]. However, these newly reported prevalence rates of $P$. aeruginosa-CAP are lower than prior reports [13-15], suggesting that only a small subgroup of patients may require empiric antipseudomonal antibiotic coverage.

The IDSA/ATS CAP guidelines recommend an antipseudomonal $\beta$-lactam antibiotic plus a fluoroquinolone or aminoglycoside in patients with risk factors for $P$. aeruginosa-CAP [13]. Dual antibiotic coverage has been recommended for HCAP, HAP or VAP where MDR Pseudomonas is thought to be an important problem [25]. Our results challenge this approach, by showing that the worldwide

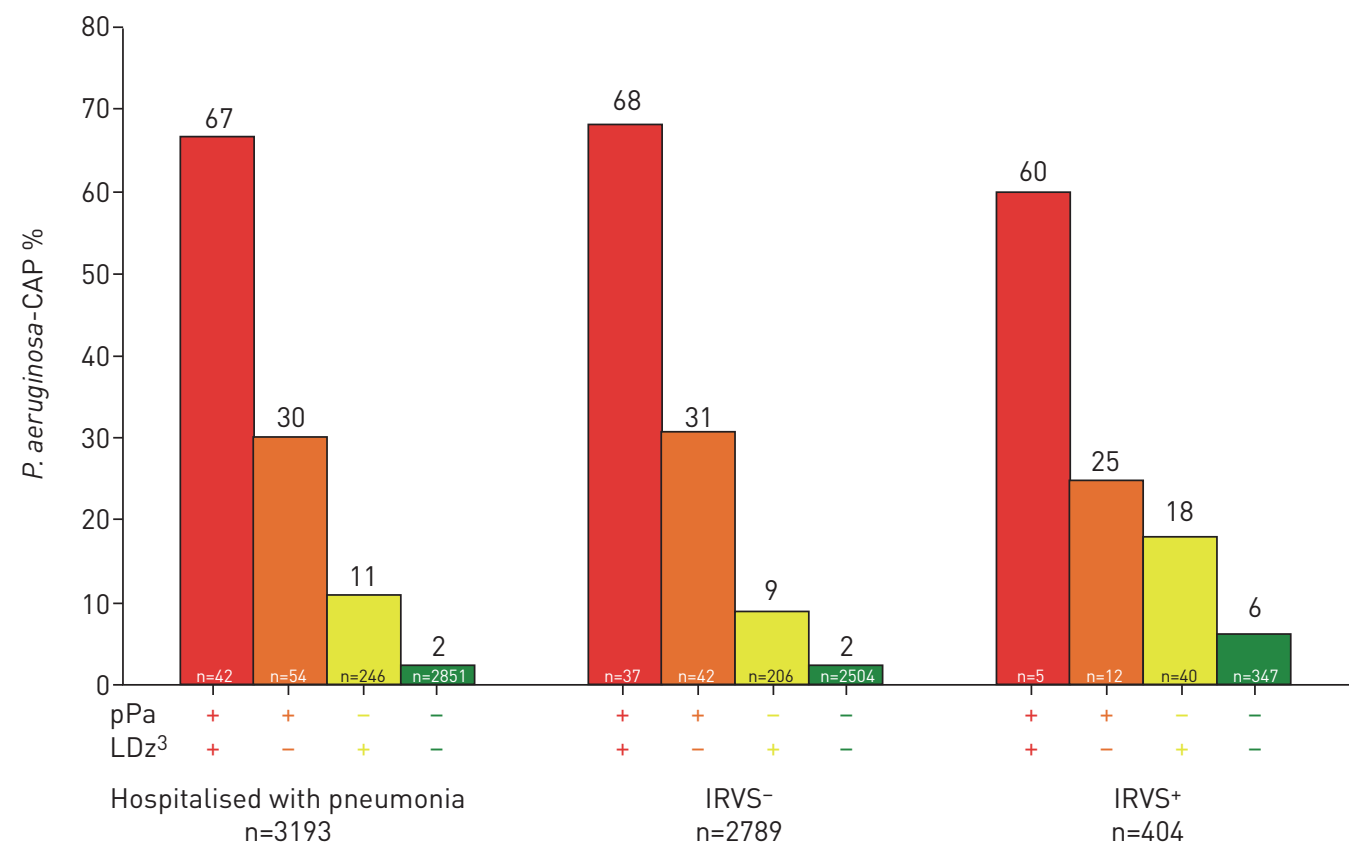

FIGURE 2 Prevalence of Pseudomonas aeruginosa-community-acquired pneumonia (CAP) according to the relevant risk factors, including prior $P$. aeruginosa (pPa) infection/colonisation, grouped variables of severe lung diseases $\left(\mathrm{LDz}^{3}\right.$ : tracheostomy, bronchiectasis and very severe chronic obstructive pulmonary disease (i.e. forced expiratory volume in $1 \mathrm{~s}<30 \%)$ ) and stratified according to the need of invasive respiratory and/or vasopressor support (IRVS). 
prevalence of MDR $P$. aeruginosa in patients with CAP is very low ( $1 \%$ or $3 \%$ among culture-positive pneumonia patients). Our results are consistent with the evidence from Spain by CillónIz et al. [33] who reported a prevalence of multidrug-resistant $P$. aeruginosa of $1.1 \%$ in a prospective cohort study of culture-positive CAP patients. Therefore, the recommended use of double antipseudomonal empirical coverage overestimates the actual rate of MDR P. aeruginosa compared to that found in infections acquired in hospital settings, where the resistance rates seems to be higher than in patients coming from the community. However, it is suggested that patients critically ill with septic shock due to pneumonia should receive empiric double antipseudomonal coverage [25].

Several researchers have hypothesised that studies assessing the prevalence of pathogens responsible for CAP are difficult to generalise, because these studies are conducted in different environments (i.e. single hospital, regional, country or continent) with specific differences in healthcare delivery, including antibiotic availability and policies for antimicrobial use [19, 21, 23, 34]. In our study, we found that the prevalence of $P$. aeruginosa-CAP was not significantly different between participating centres representing different continents. In contrast, Croatia was the only country that had statistically significant higher $P$. aeruginosa-CAP prevalence. The novelty of this study is that it enrolled patients in a large number of centres representing more than 50 countries from all over the world to identify the prevalence of $P$. aeruginosa in CAP patients.

In general, many hospitalised patients receive initial empirical antipseudomonal coverage while waiting $48-72 \mathrm{~h}$ for specific pathogen identification and antibiotic susceptibilities $[1,3]$. This practice has increased the use of broad-spectrum antibiotic agents and promoted antibiotic overuse with the risk of inducing antimicrobial resistance $[11,26]$. Additionally, the delay between initial diagnosis and availability of antibiotic susceptibilities could negatively affect outcomes in patients with CAP due to inappropriate antibiotic coverage [13]. Therefore, initiation of empiric antipseudomonal coverage should be based on the likelihood that a pathogen circulates in the affected community and the presence of specific risk factors for $P$. aeruginosa-CAP $[6,13,25]$. In this regard, the risk factors listed in the IDSA/ATS pneumonia guidelines for $P$. aeruginosa-CAP are intensive care unit admission, structural lung diseases such as bronchiectasis or COPD with multiple exacerbations [13]. Several risk factors have been reported for P. aeruginosa-CAP during the past few decades [6], but these previously reported risk factors (e.g. heart failure, tube feeding [35], etc.) were statistically significant in the bivariate analysis of our study, but not in the multivariable analysis. In contrast, we found that prior Pseudomonas infection/colonisation, prior tracheostomy, bronchiectasis, IRVS and very severe COPD (FEV1 $\leqslant 30 \%)$ were independently associated with $P$. aeruginosa-CAP. These findings support the recommendation to consider these risk factors for empirical antipseudomonal antibiotics in patients with severe pulmonary diseases, but not in all patients with structural lung diseases. More importantly, patients with previous $P$. aeruginosa infection/colonisation are at highest risk for $P$. aeruginosa-CAP and could benefit from empiric antipseudomonal antibiotic coverage, regardless of disease severity. Using these specific risk factors differs from the approach recommended by the IDSA/ATS guidelines and may help prevent overuse of antipseudomonal antibiotics in patients with CAP. More importantly, antimicrobial stewardship programmes that attempt to minimise the use of antipseudomonal coverage among patients without specific Pseudomonas risk factors may help prevent adverse events and overuse of unnecessary antimicrobial therapies.

This point prevalence study has some limitations. First, we were not able to determine clinical outcomes due to the study design. However, to determine prevalence rates and identify risk factors, point prevalence studies are ideal because they generate large sample sizes and gather generalisable data. Second, microbiological samples were not collected for centralised antibiotic susceptibility testing due to the experimental design and feasibility. Nevertheless, laboratories at study sites were encouraged to follow the CLSI or EUCAST guidelines to determine antibiotic resistance $[28,29]$. Third, microbiological diagnosis of patients with CAP could be confounded by other pathogens colonising the respiratory tract; however, in our study we only reported pathogens with representative growth according to current clinical guidelines for CAP. Fourth, infections other than pneumonia are possible, but less likely to present in patients with clinical signs and symptoms and radiological confirmation of CAP. Fifth, quality assessments were not performed because all data gathered were de-identifiable; however, all local investigators were instructed on how to enrol patients and how to use the data collection platform as described in the study protocol, data dictionary and the instructional video. Sixth, cystic fibrosis (CF) is a frequent cause of chronic $P$. aeruginosa colonisation; although the majority of patients with $\mathrm{CF}$ might be included in the bronchiectasis group, CF was not directly recorded in our study. Finally, the empirical selection of antibiotics and diagnostic approach was performed according to the healthcare provider and not according to prespecified criteria.

In conclusion, this multinational, point prevalence study found that the burden of $P$. aeruginosa, antibiotic-resistant $P$. aeruginosa and multidrug-resistant $P$. aeruginosa as the aetiologic pathogen of CAP 
is low $(<5 \%)$. We identified five risk factors associated with $P$. aeruginosa-CAP: prior Pseudomonas infection/colonisation, tracheostomy, bronchiectasis, IRVS and very severe COPD. These risk factors could serve to guide empirical antipseudomonal antibiotic treatment. Absence of these specific risk factors suggests that the empirical antibiotic treatment recommended for CAP patients will cover the most likely prevalent bacterial pathogens. Empirical antipseudomonal coverage should be reserved for a small subgroup of well-defined patients with a past medical history of Pseudomonas infection/colonisation and severe lung diseases (i.e. prior tracheostomy, bronchiectasis and/or very severe COPD), regardless of disease severity. Importantly, not all patients with chronic pulmonary diseases would require empiric antipseudomonal coverage for CAP.

Acknowledgements: We would like to thank the European Respiratory Society, the World Federation of Societies of Intensive and Critical Care Medicine, the American College of Chest Physicians, the Asociación Latinoamericana de Tórax and the Sociedad Argentina de Infectología for their support of this project.

GLIMP investigators: We would like to thank the following study contributors for their valuable collaboration: Argentina: Patricia Karina Aruj, Department of Internal Medicine, University Hospital Alfredo Lanari, Buenos Aires, Argentina; Silvia Attorri, Hospital Luis Lago Maggiore, Mendoza, Argentina; Enrique Barimboim, Hospital Central de Mendoza, Argentina; Juan Pablo Caeiro and María I. Garzón, Hospital Privado Universitario, Córdoba, Argentina; Victor Hugo Cambursano, V.H. Dr Cazaux A. Servicio de Neumologia, Hospital Rawson, Córdoba, Argentina; Adrian Ceccato, Hospital Nacional Prof Alejandro Posadas, Argentina; Julio Chertcoff, Florencia Lascar and Fernando Di Tulio, Critical Care Unit and Respiratory Medicine, Buenos Aires British Hospital, Buenos Aires, Argentina; Ariel Cordon Díaz, Hospital General Alvear, Ciudad, Mendoza, Argentina; Lautaro de Vedia, Respiratory Intensive Care Unit, Hospital Muñiz, Buenos Aires, Argentina; Maria Cristina Ganaha, Infectious Diseases Ward, Hospital Interzonal General de Agudos "Vicente Lopez y Planes" from General Rodriguez, Buenos Aires, Argentina; Sandra Lambert, Hospital El Cruce - Alta Complejidad en Red, Argentina; Gustavo Lopardo, Hospital Bernardo Houssay, Vicente López, Argentina; Carlos M. Luna, Pulmonary Medicine Division, Department of Medicine, Hospital de Clínicas, Universidad de Buenos Aires, Argentina; Alessio Gerardo Malberti, Hospital Nuestra Señora del Carmen, Argentina; Nora Morcillo and Silvina Tartara, Hospital Zonal Especializado de Agudos y Crónicos Dr Antonio A. Cetrangolo, Argentina; Claudia Pensotti, Infectious Diseases and Infection Control Department, Buenos Aires, Clinica Privada Monte Grande, Argentina; Betiana Pereyra, Hospital San Roque, Córdoba, Argentina; Pablo Gustavo Scapellato, Infectious Diseases Department, Hospital D.F. Santojanni, Argentina; Juan Pablo Stagnaro, HZGA Mi Pueblo, Florencio Varela, Argentina. Australia: Sonali Shah, Department of General Medicine, Austin Hospital, Heidelberg, Australia. Austria: Felix Lötsch and Florian Thalhammer, Division of Infectious Diseases and Tropical Medicine, Department of Medicine I, Medical University of Vienna, Austria. Belgium: Kurt Anseeuw, ZNA Campus Stuivenberg, Antwerp, Belgium; Camille A. Francois, Anesthesia and Critical Care Department, Erasme University Hospital, Brussels, Belgium; Eva Van Braeckel, Department of Respiratory Medicine, Ghent University Hospital, Belgium; Jean Louis Vincent, Department of Intensive Care, Erasme University Hospital, Université Libre de Bruxelles, Brussels, Belgium. Benin: Marcel Zannou Djimon, Jules Bashi and Roger Dodo, Centre Hospitalier Universitaire HKM of Cotonou, Benin. Brazil: Simone Aranha Nouér, Federal University of Rio de Janeiro, Brazil. Bulgaria: Peter Chipev and Milena Encheva, Clinic of Pulmonary Diseases, Military Medical Academy, Sofia, Bulgaria; Darina Miteva, UMHAT "St. Marina", Varna, Bulgaria; Diana Petkova, University Hospital Varna, Bulgaria. Cameroon: Adamou Dodo Balkissou, Yaounde Jamot Hospital, Yaounde, Cameroon; Eric Walter Pefura Yone, Département de Médecine Interne, University of Yaounde, Yaoundé, Cameroon; Bertrand Hugo Mbatchou Ngahane, Douala General Hospital, Douala, Cameroon. China: Ning Shen, Respiratory Medicine, Peking University Third Hospital, Beijing, China; Jin-fu Xu, Department of Respiratory Medicine, Shanghai Pulmonary Hospital, Tongji University, China. Colombia: Carlos Andres Bustamante Rico and Ricardo Buitrago, Clinica Shaio, Bogota, Colombia; Fernando Jose Pereira Paternina, Las Americas Clinic, Medellin, Colombia. Congo: Jean-Marie Kayembe Ntumba, Cliniques Universitaires de Kinshasa, DR Congo. Croatia: Vesna Vladic Carevic, Interne Medicine, Dubrovnik, Croatia; Marko Jakopovic, Medical School, University of Zagreb, Department for Respiratory Diseases Jordanovac, University Hospital Centre Zagreb, Zagreb, Croatia; Mateja Jankovic, University Hospital Center Zagreb, Department for Respiratory Diseases, Zagreb, Croatia; Zinka Matkovic, University Hospital Dubrava, Zagreb, Croatia; Ivan Mitrecic, Karlovac General Hospital, Karlovac, Croatia. Denmark: Marie-Laure Bouchy Jacobsson, Emergency Department in North Zealand's Hospital, Hillerød, Denmark; Anette Bro Christensen, Department of Anaethesiology, Viborg Region Hospital, Denmark; Uffe Christian Heitmann Bødtger, Department of Pulmonology, Naestved Hospital, Denmark; Christian Niels Meyer, Department of Internal Medicine, Roskilde Hospital, Copenhagen University Hospital, Roskilde, Denmark; Andreas Vestergaard Jensen, Gertrud Baunbæk-Knudsen, Pelle Trier Petersen and Stine Andersen, Department of Lung- and Infectious Diseases, Nordsjællands Hospital-Hillerød, Denmark. Egypt: Ibrahim El-Said Abd El-Wahhab, Thoracic Medicine, Faculty of Medicine - Mansoura University, Egypt; Nesreen Elsayed Morsy, Pulmonary, Critical Care and Sleep Medicine, Faculty of Medicine, Mansoura University, Mansoura, Egypt; Hanaa Shafiek, Chest Diseases Department, Faculty of Medicine, Alexandria University, Egypt; Eman Sobh, Chest Diseases Department, Al-Azhar University, Cairo, Egypt. Ethiopia: Kedir Abdella Abdulsemed, Department of Medical Laboratory Science and Pathology, College of Health Sciences, Mycobacteriology Research Centre, Institute of Biotechnology Research, Jimma University, Jimma, Ethiopia. France: Fabrice Bertrand, Critical Care Unit, Robert Ballanger Hospital, Aulnay sous Bois, France; Christian Brun-Buisson, Univ Hospital Henri Mondor, 94000 Créteil, France; Etienne de Montmollin, Intensive Care Unit, Hôpital Delafontaine, Centre hospitalier de Saint-Denis, Saint-Denis, France; Muriel Fartoukh, Unité de réanimation médico-chirurgicale, Pôle Thorax Voies aériennes, Hôpital Tenon, Groupe Hospitalier Est Parisien, France; Jonathan Messika, Publique-Hôpital de Paris, Service de Réanimation Médico-chirurgicale, Hôpital Louis Mourier, Colombes, France and Université Paris Diderot, IAME, UMR 1137, Sorbonne Paris Cité, Paris, France; Pierre Tattevin, Infectious Diseases and ICU, Pontchaillou University Hospital, Rennes, France; Abdo Khoury, Department of Emergency Medicine and Critical Care, University of Franche - Comté, Medical Center, France. Gambia: Bernard Ebruke, Medical Research Council Unit, Gambia. Germany: Michael Dreher, Department of Cardiology, Pneumology, Vascular Medicine and Intensive Care Medicine, University Hospital Aachen, Aachen, Germany; Martin Kolditz, Division of Pulmonology, Medical Department I, University Hospital Carl Gustav Carus, Technische Universität 
Dresden, Germany; Matthias Meisinger, Klinikum Niederlausitz GmbH, Klinik für Innere Medizin und Intensivmedizin, Senftenberg, Germany; Mathias W. Pletz and Stefan Hagel, Center for Infectious Diseases and Infection Control, Jena University Hospital, Germany; Jan Rupp, Department of Molecular and Infectious Diseases, University of Lübeck, Lübeck, Germany; Tom Schaberg, Zentrum für Pneumologie, Agaplesion Diakonieklinikum Rotenburg, Germany; Marc Spielmanns, Internal Medicine Department, Pulmonary Rehabilitation and Department of Health, School of Medicine, University Witten-Herdecke, St. Remigius-Hospital, Leverkusen, Germany; Petra Creutz and Norton Suttorp, Department of Infectious Disease and Respiratory Medicine, Charité - University Medicine, Berlin, Germany. Ghana: Beatrice Siaw-Lartey, Komfo-Anokye Teaching Hospital, Kumasi, Ghana. Greece: Katerina Dimakou, 5th Respiratory Medicine Dpt, "SOTIRIA" Chest Hospital, Athens 11527, Greece; Dimosthenis Papapetrou, Medical Group of Athens (Paleo Faliro Clinic), Athens, Greece; Evdoxia Tsigou and Dimitrios Ampazis, Agioi Anargiroi Hospital, Kifissia, Athens, Greece; Evangelos Kaimakamis, Intensive Care Unit, "G. Papanikolaou" General Hospital of Thessaloniki, Greece. India: Mohit Bhatia, S.S. Hospital IMS BHU Varanasi, India; Raja Dhar, Fortis Hospitals, Kolkata, India; George D'Souza, Department of Pulmonary Medicine, St John's Medical College Hospital, Bangalore, India; Rajiv Garg, Department of Respiratory Medicine, King George's Medical University UP, Lucknow, India; Parvaiz A. Koul, Department of Internal and Pulmonary Medicine, SheriKashmir Institute of Medical Sciences, Srinagar, India; P.A. Mahesh and B.S. Jayaraj, Department of Pulmonary Medicine, JSS Medical College, JSS University, Mysore, India; Kiran Vishnu Narayan, Pulmonary Medicine, Government Medical College Kozhikode, Kerala, India; Hirennappa B. Udnur and Shashi Bhaskara Krishnamurthy, Columbia Asia Hospital, Hebbal, Bengaluru, Karnataka, India; Surya Kant, Department of Respiratory Medicine, King George's Medical University, Chowk, Lucknow, India; Rajesh Swarnakar, Getwell Hospital and Research Institute, Dhantoli, Nagpur, India; Sneha Limaye and Sundeep Salvi, on behalf of the Respiratory Research Network of India (RRNI) from the Chest Research Foundation in Pune, India. Iran: Keihan Golshani, Isfahan University of Medical Sciences, Iran. Ireland: Vera M. Keatings, Letterkenny General Hospital, Co. Donegal, Ireland; Ignacio Martin-Loeches, Multidisciplinary Intensive Care Research Organization (MICRO), St James's University Hospital, Trinity Centre for Health Sciences Dublin, Ireland. Israel: Yasmin Maor, Infectious Disease Unit, Affiliated to Tel Aviv University, Wolfson Medical Center, Holon, Israel; Jacob Strahilevitz, Department of Clinical Microbiology and Infectious Diseases, Hadassah-Hebrew University, Jerusalem, Israel. Italy: Salvatore Battaglia, University of Palermo, Pneumologia DiBiMIS, Palermo, Italy; Maria Carrabba, Internal Medicine Department, Fondazione IRCCS Ca' Granda Ospedale Maggiore Policlinico, 20122, Milano, Italy; Piero Ceriana, Pulmonary Rehabilitation, IRCCS Fondazione Maugeri, 27100, Pavia, Italy; Marco Confalonieri, Department of Pulmunology, University Hospital, Trieste, Italy; Antonella d'Arminio Monforte, Department of Health Sciences, Clinic of Infectious Disease, San Paolo Hospital, University of Milan, Italy; Bruno Del Prato, Interventional Pneumology, Hospital Antonio Cardarelli, Naples, Italy; Marino De Rosa, UOC Pneumologia P.O. San Filippo Neri ASL RM E Roma, Italy; Riccardo Fantini, Respiratory Diseases clinic, Policlinico di Modena, 41124 Modena, Italy; Giuseppe Fiorentino, UOC Fisiopatologia e Riabilitazione Respiratoria AO Ospedali dei Colli PO Monaldi, Italy; Maria Antonia Gammino, Pulmonary Medicine Unit, San Martino Hospital, ASL 5 Oristano, Sardegna, Italy; Francesco Menzella, Department of Cardiac-Thoracic-Vascular and Intensive Care Medicine, Pneumology Unit, IRCCS-Arcispedale Santa Maria Nuova, Reggio Emilia, Italy; Giuseppe Milani, Azienda Ospedaliera Sant Anna di Como, Presidio Ospedale S. Anna Nuovo, Unità Operativa di Pneumologia, Como, Italy; Stefano Nava, Alma Mater University of Bologna, DIMES, Respiratory and Critical Care Unit Sant'Orsola Malpighi Hospital, Italy; Gerardo Palmiero, Respiratory Unit, Versilia Hospital, Azienda USL 12 Viareggio, Lido di Camaiore, Lucca, Italy; Roberta Petrino and Barbra Gabrielli, Emergency Medicine Unit, S. Andrea Hospital, Vercelli, Italy; Paolo Rossi, Internal Medicine Department, Azienda Ospedaliero-Universitaria S. Maria della Misericordia, Udine, Italy; Claudio Sorino, Pulmonology Unit, A.O. Sant'Anna di Como, Italy; Gundi Steinhilber, Spedali Civili Brescia, U.O. Pneumologia e Fisiopatologia Respiratoria, Brescia, Italy; Alessandro Zanforlin, ULSS 18 Rovigo, Ospedale San Luca, 45027 Trecenta (RO), Italy; Fabio Franzetti, Manuela Carugati, Manuela Morosi and Elisa Monge, Department of Biomedical and Clinical Sciences, Division of Infectious Diseases, Luigi Sacco Hospital, Università degli Studi di Milano, Milan, Italy; Mauro Carone, Fondazione Salvatore Maugeri, IRCCS, Cassano Murge, Italy; Vincenzo Patella, Allergology and Clinical Immunology Unit, Department of Medical Sciences, Battipaglia Hospital, Battipaglia, Salerno, Italy; Simone Scarlata, Geriatrics, Unit of Respiratory Pathophysiology and Thoracic Endoscopy, Campus Bio Medico University and Teaching Hospital, Rome, Italy; Andrea Comel, UO Pneumologia, Ospedale Pederzoli, Peschiera del Garda, Italy. Japan: Kiyoyasu Kurahashi, Yokohama City University Medical Center, Japan. Lebanon: Zeina Aoun Bacha, Medicine School, St Joseph University, Beyrouth, Lebanon. Mexico: Daniel Barajas Ugalde, National Institute of Respiratory Diseases, Mexico; Omar Ceballos Zuñiga, Hospital General de Mexicali, Mexicali, Baja California, Mexico; José F. Villegas, Hospital Universitario Monterrey, n. l. México CP 64030. Montenegro: Milic Medenica, Hospital for Lung Diseases - Brezovik, Niksic, Montenegro. Nepal: Deebya Raj Mihsra, Internal Medicine, BP Koirala Institute of Health Sciences, Nepal; Poojan Shrestha, Oxford University Clinical Research Unit, Patan Hospital, Nepal. The Netherlands: E.M.W. van de Garde, Dept Clinical Pharmacy, St Antonius Hospital, Utrecht/Nieuwegein, The Netherlands. New Zealand: Elliott Ridgeon, Medical Research Institute of New Zealand. Nigeria: Babatunde Ishola Awokola, Department of Family Medicine and Primary Care, Lily Hospitals Limited, Warri, Nigeria; Ogonna N.O. Nwankwo, University of Calabar Teaching Hospital, Calabar, Nigeria; Adefuye Bolanle Olufunlola, Olabisi Onabanjo University Teaching Hospital, Sagamu, Ogun State, Nigeria; Segaolu Olumide, Department of Medicine (Pulmonary Unit), University College Hospital, Ibadan, Nigeria; Kingsley N. Ukwaja, Department of Medicine, Federal Teaching Hospital Abakaliki, Ebonyi State, Nigeria. Pakistan: Muhammad Irfan, Section of Pulmonary and Critical Care Medicine, Department of Medicine, Aga Khan University, Karachi-74800, Pakistan. Poland: Lukasz Minarowski, Department of Lung Diseases and Tuberculosis, Medical University of Bialystok, Poland; Skoczyński Szymon, Department of Pneumology, School of Medicine in Katowice, Medical University of Silesia, Katowice, Institute of Occupational Medicine and Environmental Health, Sosnowiec, Poland. Portugal: Felipe Froes, Hospital Pulido Valente - CHLN, Lisboa, Portugal; Pedro Leuschner, Centro Hospitalar do Porto, Porto, Portugal; Mariana Meireles, Cláudia Ferrão, Pedro Leuschner and João Neves, Serviço de Medicina, Centro Hospitalar do Porto, Largo Prof. Abel Salazar, 4099-001 Porto, Portugal; Sofia B Ravara, Faculty of Health Sciences, University of Beira Interior; Cova da Beira Hospital Center, 6200-251 Covilhã, Portugal. Republic of Moldova: Victoria Brocovschii, Department of Pneumology and Allergology, State University of Medicine and Pharmacy "Nicolae Testemitanu" Republic of Moldova; Chesov Ion, Clinic of Anesthesia and Intensive Care "Valeriu Ghrerg", Institute of Emergency Medicine, State University of Medicine and Pharmacy "Nicolae Testemitanu", Chisinau, Republic of Moldova; Doina Rusu, SMFU "N. Testemitanu”, Chisinau, Republic of Moldova; Cristina Toma, Department of Pneumology and 
Allergology, State University of Medicine and Pharmacy "Nicolae Testemitanu", Chisinau, Republic of Moldova. Romania: Daniela Chirita, Hospital Sfantul Stefan, Bucharest, Romania; Carmen Mihaela Dorobat, Universitatea de Medicină şi Farmacie "Gr. T. Popa” Iaşi Facultatea de Medicină Stomatologică, Spitalul Clinic de Boli Infecțioase "Sfânta Parascheva", Iași, Romania. Russia: Alexei Birkun, Department of Anesthesiology, Critical Care and Emergency Medicine, Medical Academy named after S. I. Georgievsky, Russian Federation; Anna Kaluzhenina, Volgograd State Medical University, Russia. Saudi Arabia: Abdullah Almotairi, King Fahad Medical City (KFMC), Riyadh, KSA; Zakeya Abdulbaqi Ali Bukhary, College of Medicine, Taibah University, Medina, KSA; Jameela Edathodu, Al Faisal University, King Faisal Specialist Hospital, Riyadh, KSA; Amal Fathy, Pulmonary and Respiratory Critical Care Medicine, Mansoura University Egypt, Affiliate at Taibah University, KSA; Abdullah Mushira Abdulaziz Enani and Nazik Eltayeb Mohamed, Infectious Diseases Section, Medical Specialties Department, King Fahad Medical City, Riyadh, KSA; Jawed Ulhadi Memon, Pulmonology Division, Department of Internal Medicine, King Fahad Hospital, Hofuf, Al Ahasa, 31982, KSA; Abdelhaleem Bella, Dammam University-Saudi Arabia and King Fahad Hospital, KSA. Serbia: Nada Bogdanović, Pulmonary Department of KHC Dr. Dragiša Mišović, Belgrade, Serbia; Branislava Milenkovic, Clinic for Pulmonary Diseases, Clinical Centre of Serbia, Faculty of Medicine, University of Belgrade, Belgrade, Serbia; Dragica Pesut, University of Belgrade School of Medicine, Teaching Hospital of Pulmonology, Clinical Centre of Serbia, Belgrade, Serbia. South Africa: Charles Feldman, Division of Pulmonology, Department of Internal Medicine, Charlotte Maxeke Johannesburg Academic Hospital, Faculty of Health Sciences, University of the Witwatersrand, Johannesburg, South Africa. South Korea: Ho Kee Yum, Inje Univ. Seoul Paik Hospital, South Korea. Spain: Luis Borderìas, Respiratory and Sleep Unit, Hospital San Jorge, Huesca, Spain; Noel Manuel Bordon Garcia, Barcelona Policlínic and Moises Broggi Hospital at sant Joan Despí, Spain; Hugo Cabello Alarcón, Sant Hospital Seu de Urgell, Catalonia, Spain; Catia Cilloniz and Antoni Torres, Department of Pneumology, Institut Clinic del Tórax, Hospital Clinic of Barcelona, Institut d'Investigacions Biomèdiques August Pi i Sunyer (IDIBAPS), University of Barcelona, Spain; Vicens Diaz-Brito and Xavier Casas, Infectious Diseases Unit and Pneumology Service, Parc Sanitari Sant Joan de Deu, Sant Boi, Barcelona, Spain; Alicia Encabo González, Hospital Complex of Pontevedra, Spain; Maria Luisa Fernández-Almira, Medicina Interna, Hospital Universitario Central de Asturias, Spain; Miguel Gallego, Department of Respiratory Medicine, Hospital de Sabadell, Institut Universitari Parc Taulí-UAB, Sabadell, CIBER de Enfermedades Respiratorias, CIBERES, Bunyola, Spain; Inmaculada Gaspar-García, Department of Respiratory Medicine, Hospital Costa del Sol, Marbella, Málaga, Spain; Juan González del Castillo, Emergency Department, Hospital Universitario Clínico San Carlos, Madrid, Spain; Patricia Javaloyes Victoria, Hospital General Universitario de Alicante, Alicante, Spain; Elena Laserna Martínez, Hospital Mollet, Barcelona, Spain; Rosa Malo de Molina, University Hospital Puerta de Hierro Majadahonda, Madrid, Spain; Pedro J. Marcos, Servicio de Neumología, Complejo Hospitalario Universitario de A Coruña (CHUAC), INIBIC, Sergas, Universidade de A Coruña (UDC), Spain; Rosario Menéndez, Pneumology Service, Universitary and Polytechnic Hospital La Fe, Valencia, Spain; Ana Pando-Sandoval, Hospital Universitario Central de Asturias. Area de Gestion Clinica de Pulmon. Servicio de Neumologia, Oviedo, Spain; Cristina Prat Aymerich, Alicia Lacoma de la Torre and Ignasi García-Olivé, Microbiology Department and Pneumology Department, Hospital Universitari Germans Trias i Pujol, Institut d'Investigació Germans Trias i Pujol, Badalona, Universitat Autònoma de Barcelona, CIBER Enfermedades Respiratorias (CIBERES), Instituto de Salud Carlos III, Spain; Jordi Rello and Silvia Moyano, Critical Care Department, Hospital Vall d'Hebron, Barcelona, Spain; Francisco Sanz, Servicio de Neumología, Consorci Hospital General Universitari de Valencia, Valencia, Spain; Oriol Sibila and Ana Rodrigo-Troyano, Servei de Pneumologia, Hospital de la Santa Creu i Sant Pau, IIB-Sant Pau, Barcelona, Spain; Jordi Solé-Violán, Hospital Universitario de Gran Canaria Dr Negrín, Las Palmas de Gran Canaria, Spain; Ane Uranga, Pulmology Department, Hospital of Galdakao-Usansolo, Spain; Job F.M. van Boven, Hospital Universitari Son Espases, Palma de Mallorca, Spain; Ester Vendrell Torra and Jordi Almirall Pujol, Intensive Care Medicine, Hospital de Mataró, Spain. Togo: Arnauld Attannon Fiogbe, Pulmonology and Infectious Diseases Service/University Hospital of Sylvanus Olympio, Lomé, Togo. Tunisia: Ferdaous Yangui, Department of Pneumology, Hospital of Internal Forces Security (I.F.S), Marsa, Tunis, Tunisia. Turkey: Semra Bilaceroglu, Izmir Dr. Suat Seren Training and Research Hospital for Thoracic Medicine and Surgery, Izmir, Turkey; Levent Dalar, Pulmonary Medicine, Istanbul Bilim University, Istanbul, Turkey; Ufuk Yilmaz, Suat Seren Chest Disease and Surgery Training and Research Hospital, İzmir, Turkey. Ukraine: Artemii Bogomolov, Vinnitsa National Pirogov Memorial Medical University, Vinnitsa Regional Antituberculosis Hospital, Vinnitsa, Ukraine. United Arab Emirates: Naheed Elahi, Dubai Hospital, UAE. UK: Devesh J. Dhasmana, Victoria Hospital, Kirkcaldy, NHS Fife, UK; Andrew Feneley, Rhiannon Ions, Julie Skeemer and Gerrit Woltmann, University Hospitals of Leicester NHS Trust and University of Leicester, Leicester, UK; Carole Hancock, Royal Respiratory Research Team, Royal Liverpool University Hospital, Liverpool, UK; Adam T. Hill, Royal Infirmary and University of Edinburgh, UK; Banu Rudran, The Royal London Hospital, Barts Health Trust, London, UK; Silvia Ruiz-Buitrago and Marion Campbell, Hairmyres Hospital, East Kilbride, UK; Paul Whitaker, Department of Respiratory Medicine, St James's Hospital, Leeds, UK; Alexander Youzguin, Southport and Ormskirk Hospitals NHS Trust, UK; Anika Singanayagam, Imperial College Healthcare NHS Trust, London, UK. USA: Karen S. Allen, University of Oklahoma Health Sciences Center, USA; Veronica Brito, Texas A\&M Health Science Center, Division of Pulmonary, Critical Care and Sleep Medicine Baylor Scott and White Health, USA; Jessica Dietz, Fargo VA Health Care System, Fargo, North Dakota, USA; Claire E. Dysart and Susan M. Kellie, Clement J. Zablocki VA Medical Center, Milwaukee, WI, USA, Division of Infectious Diseases, University of New Mexico School of Medicine, Raymond G. Murphy VA Medical Center, Albuquerque, NM, USA; Ricardo A. Franco-Sadud and Garnet Meier, Division of Hospital Medicine, Cook County Hospital, Chicago, USA; Mina Gaga, 7th Resp. Med. Dept and Asthma Center, Athens Chest Hospital, USA; Thomas L. Holland and Stephen P. Bergin, Department of Medicine, Duke University Medical Center and School of Medicine, Duke Clinical Research Institute, USA; Fayez Kheir, Department of Pulmonary Diseases, Critical Care and Environmental Medicine, Tulane University Health Sciences Center, New Orleans, LA, USA; Mark Landmeier, Division of Pulmonary and Critical Care Medicine, Northwestern Memorial Hospital, Chicago, IL, USA; Manuel Lois, John Peter Smith Hospital, Fort Worth, TX, USA; Girish B. Nair, Interstitial Lung Disease Program and Pulmonary Rehabilitation, SUNY Stony Brook Winthrop University Hospital, Mineola, NY, USA; Hemali Patel, Department of Medicine, Division of General Internal Medicine, Hospital Medicine Group, University of Colorado, USA; Katherine Reyes, Henry Ford Hospital, Detroit, IL, USA; William Rodriguez-Cintron, Pulmonary/Critical Care Medicine VA Caribbean Healthcare System, USA; Shigeki Saito, Tulane University, New Orleans, USA; Nilam J. Soni, Julio Noda, Cecilia I. Hinojosa, Stephanie M. Levine, Luis F. Angel and Antonio Anzueto, Divisions of Hospital Medicine and Pulmonary/Critical Care Medicine, South Texas Veterans Health Care System, University of Texas Health Science Center San Antonio, San Antonio, TX, USA; K. Scott 
Whitlow, John Hipskind, Kunal Sukhija and Vicken Totten, Kaweah Delta Health Care District, Department of Emergency Medicine, Visalia, CA, USA; Richard G. Wunderink and Ray D. Shah, Northwestern University Feinberg School of Medicine, Chicago, IL, USA. Zambia: Kondwelani John Mateyo, Department of Internal Medicine, University Teaching Hospital, Lusaka, Zambia. Other investigators: Lorena Noriega; Ezequiel Alvarado; Mohamed Aman; Lucía Labra.

Conflict of interest: J.D. Chalmers has received research grants for COPD studies and personal fees from GlaxoSmithKline, Boehringer Ingelheim and Pfizer, research grants for COPD studies from AstraZeneca, research grants for bronchiectasis studies and personal fees from Bayer Healthcare and Grifols, and personal fees for consultancy from Napp, outside the submitted work. S. Aliberti reports grants and personal fees from Bayer Healthcare, Aradigm Corporation, Grifols, Chiesi and INSMED, and personal fees from AstraZeneca, Basilea, Zambon, Novartis, Raptor, Actavis UK Ltd and Horizon, outside the submitted work.

Support statement: This project was unfunded. However, Nilam Soni's time is partially funded by the Department of Veterans Affairs, Quality Enhancement Research Initiative (QUERI) Partnered Evaluation Initiative Grant (HX002263-01A1). The content is solely the responsibility of the authors and does not necessarily represent the official views of the Department of Veterans Affairs, nor the United States Government.

\section{References}

$1 \quad$ Prina E, Ranzani OT, Torres A. Community-acquired pneumonia. Lancet 2015; 386: 1097-1108.

2 World Health Organization. The Top 10 Causes of Death. www.who.int/news-room/fact-sheets/detail/ the-top-10-causes-of-death

3 Wunderink RG, Waterer GW. Community-acquired pneumonia. N Engl J Med 2014; $370: 1863$.

4 Musher DM, Thorner AR. Community-acquired pneumonia. N Engl J Med 2014; 371: 1619-1628.

5 Jain S, Self WH, Wunderink RG, et al. Community-acquired pneumonia requiring hospitalization among U. S. adults. N Engl J Med 2015; 373: 415-427.

6 Sibila O, Rodrigo-Troyano A, Shindo Y, et al. Multidrug-resistant pathogens in patients with pneumonia coming from the community. Curr Opin Pulm Med 2016; 22: 219-226.

7 Palacio F, Reyes LF, Levine DJ, et al. Understanding the concept of health care-associated pneumonia in lung transplant recipients. Chest 2015; 148: 516-522.

8 Polverino E, Torres A, Menendez R, et al. Microbial aetiology of healthcare associated pneumonia in Spain: a prospective, multicentre, case-control study. Thorax 2013; 68: 1007-1014.

9 Sibila O, Restrepo MI, Anzueto A. What is the best antimicrobial treatment for severe community-acquired pneumonia (including the role of steroids and statins and other immunomodulatory agents). Infect Dis Clin North Am 2013; 27: 133-147.

10 Wunderink RG, Yin Y. Antibiotic resistance in community-acquired pneumonia pathogens. Semin Respir Crit Care Med 2016; 37: 829-838.

11 Chalmers JD, Reyes LF, Aliberti S, et al. Empirical coverage of methicillin-resistant Staphylococcus aureus in community-acquired pneumonia: those who do not remember the past are doomed to repeat it. Clin Infect Dis 2016; 63: 1145-1146

12 Lister PD, Wolter DJ, Hanson ND. Antibacterial-resistant Pseudomonas aeruginosa: clinical impact and complex regulation of chromosomally encoded resistance mechanisms. Clin Microbiol Rev 2009; 22: 582-610.

13 Mandell LA, Wunderink RG, Anzueto A, et al. Infectious Diseases Society of America/American Thoracic Society consensus guidelines on the management of community-acquired pneumonia in adults. Clin Infect Dis 2007; 44: Suppl. 2, S27-S72.

14 Brito V, Niederman MS. Healthcare-associated pneumonia is a heterogeneous disease, and all patients do not need the same broad-spectrum antibiotic therapy as complex nosocomial pneumonia. Curr Opin Infect Dis 2009; 22: 316-325.

15 Kollef MH, Shorr A, Tabak YP, et al. Epidemiology and outcomes of health-care-associated pneumonia: results from a large US database of culture-positive pneumonia. Chest 2005; 128: 3854-3862.

16 Restrepo MI, Anzueto A. The role of Gram-negative bacteria in healthcare-associated pneumonia. Semin Respir Crit Care Med 2009; 30: 61-66.

17 Arancibia F, Bauer TT, Ewig S, et al. Community-acquired pneumonia due to Gram-negative bacteria and Pseudomonas aeruginosa: incidence, risk, and prognosis. Arch Intern Med 2002; 162: 1849-1858.

18 Sibila O, Laserna E, Maselli DJ, et al. Risk factors and antibiotic therapy in P. aeruginosa community-acquired pneumonia. Respirology 2015; 20: 660-666.

19 Waterer GW. Healthcare-associated pneumonia: can we salvage anything from the wreckage? Respirology 2016; 21: 8-9.

20 Torres A, Cillóniz C, Ferrer M, et al. Bacteraemia and antibiotic-resistant pathogens in community acquired pneumonia: risk and prognosis. Eur Respir J 2015; 45: 1353-1363.

21 Ewig S, Welte T, Chastre J, et al. Rethinking the concepts of community-acquired and health-care-associated pneumonia. Lancet Infect Dis 2010; 10: 279-287.

22 Lopez A, Amaro R, Polverino E. Does health care associated pneumonia really exist? Eur J Intern Med 2012; 23: 407-411.

23 Restrepo MI, Aliberti S. Healthcare-associated pneumonia: where do we go next? Clin Infect Dis 2014; 58: 340-341.

24 Chalmers JD, Rother C, Salih W, et al. Healthcare-associated pneumonia does not accurately identify potentially resistant pathogens: a systematic review and meta-analysis. Clin Infect Dis 2014; 58: 330-339.

25 Kalil AC, Metersky ML, Klompas M, et al. Executive summary: management of adults with hospital-acquired and ventilator-associated pneumonia: 2016 clinical practice guidelines by the Infectious Diseases Society of America and the American Thoracic Society. Clin Infect Dis 2016; 63: 575-582.

26 Aliberti S, Reyes LF, Faverio P, et al. Global initiative for meticillin-resistant Staphylococcus aureus pneumonia (GLIMP): an international, observational cohort study. Lancet Infect Dis 2016; 16: 1364-1376. 
27 Harris PA, Taylor R, Thielke R, et al. Research electronic data capture (REDCap) - a metadata-driven methodology and workflow process for providing translational research informatics support. $J$ Biomed Inform 2009; 42: 377-381.

28 Clinical and Laboratory Standards Institute (CSLI). Performance Standards for Antimicrobial Susceptibility Testing; Twenty-Fifth Informational Supplement. Wayne, CLSI, 2015.

29 Hsueh PR, Ko WC, Wu JJ, et al. Consensus statement on the adherence to Clinical and Laboratory Standards Institute (CLSI) Antimicrobial Susceptibility Testing Guidelines (CLSI-2010 and CLSI-2010-update) for Enterobacteriaceae in clinical microbiology laboratories in Taiwan. J Microbiol Immunol Infect 2010; 43: 452-455.

30 Charles PG, Wolfe R, Whitby M, et al. SMART-COP: a tool for predicting the need for intensive respiratory or vasopressor support in community-acquired pneumonia. Clin Infect Dis 2008; 47: 375-384.

31 Magiorakos AP, Srinivasan A, Carey RB, et al. Multidrug-resistant, extensively drug-resistant and pandrugresistant bacteria: an international expert proposal for interim standard definitions for acquired resistance. Clin Microbiol Infect 2012; 18: 268-281.

32 Chalmers JD, Taylor JK, Singanayagam A, et al. Epidemiology, antibiotic therapy, and clinical outcomes in health care-associated pneumonia: a UK cohort study. Clin Infect Dis 2011; 53: 107-113.

33 Cillóniz C, Ewig S, Polverino E, et al. Microbial aetiology of community-acquired pneumonia and its relation to severity. Thorax 2011; 66: 340-346.

34 Aliberti S, Cilloniz C, Chalmers JD, et al. Multidrug-resistant pathogens in hospitalised patients coming from the community with pneumonia: a European perspective. Thorax 2013; 68: 997-999.

35 von Baum $\mathrm{H}$, Welte $\mathrm{T}$, Marre $\mathrm{R}$, et al. Community-acquired pneumonia through Enterobacteriaceae and Pseudomonas aeruginosa: diagnosis, incidence and predictors. Eur Respir J 2010; 35: 598-605. 